\title{
Chlamydia trachomatis infection in women attending health centres in Babol: prevalence and risk factors
}

\author{
A. Bakhtiari ${ }^{1}$ and A. Firoozjahi
}

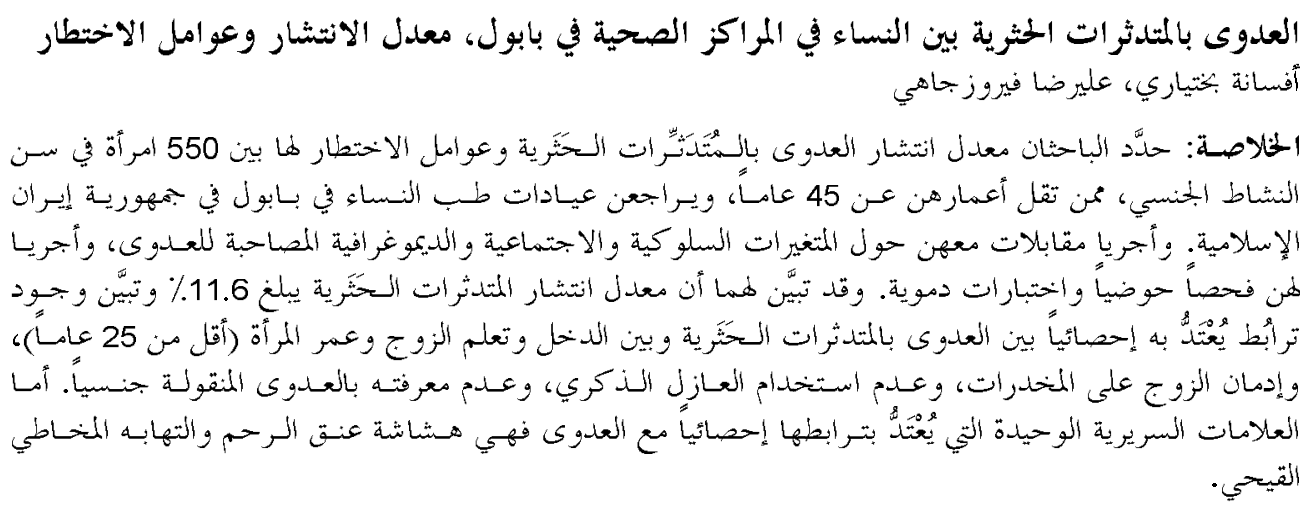

ABSTRACT We determined the prevalence of Chlamydia trachomatis infection and risk factors for infection among 550 sexually active women $<45$ years attending gynaecology clinics in Babol, Islamic Republic of Iran. The women were interviewed about sociodemographic and behavioural variables associated with the infection and underwent pelvic examination and blood tests. The prevalence of $C$. trachomatis was $11.6 \%$. There was a significant association between $C$. trachomatis infection and income, husband's education and woman's age ( $<25$ years), and also drug addiction of husband, non-use of condoms and not having heard of sexually transmitted infections. The only clinical signs significantly associated with infection were cervical friability and mucopurulent cervicitis.

L'infection à Chlamydia trachomatis chez les femmes consultant les centres de santé de Babol : prévalence et facteurs de risque

RÉSUMÉ Nous avons déterminé la prévalence de l'infection à Chlamydia trachomatis et les facteurs de risque infectieux chez 550 femmes sexuellement actives, âgées de moins de 45 ans et accueillies aux consultations de gynécologie de la ville de Babol en République islamique d'Iran. Ces femmes ont été soumises à un interrogatoire portant sur les variables sociodémographiques et comportementales liées à l'infection et ont subi un examen gynécologique, ainsi que des tests sanguins. Nous avons constaté une prévalence de $11,6 \%$ de $C$. trachomatis. II est apparu une association significative entre l'infection à C. trachomatis et, d'une part, le revenu du foyer, le niveau d'instruction de l'époux et l'âge de la femme (<25 ans) et d'autre part, la toxicomanie du conjoint, la non-utilisation de préservatifs et l'ignorance de l'existence des infections sexuellement transmissibles. La friabilité du col utérin et la cervicite mucopurulente sont les seuls signes associés de manière significative à l'infection.

${ }^{1}$ Department of Midwifery; ${ }^{2}$ Department of Pathology, Babol University of Medical Sciences, Babol, Islamic Republic of Iran (Correspondence to A. Bakhtiari: a.bakhtiari@mubabol.ac.ir).

Received: 19/06/05; accepted: 22/09/05 


\section{Introduction}

Most sexually transmitted infections (STIs) caused by bacteria have been declining in industrialized countries since 1980 , but they are of major public health concern in developing countries [1]. Of more than 20 types of STIs, genital Chlamydia trachomatis (CT) and Neisseria gonorrhoeae are the most prevalent throughout the world [2]. Worldwide, there is an estimated annual incidence of 50 million cases of CT [3]. The magnitude of morbidity associated with this infection is enormous. It is a common cause of urethritis and cervicitis. Sequelae include pelvic inflammatory disease (PID), ectopic pregnancy, tubal factor infertility, epididymitis, proctitis and reactive arthritis. The sharp worldwide increase in the incidence of PID over the past 2 decades has led to secondary epidemics of tubal factor infertility and ectopic pregnancy. Chlamydial PID is the most important preventable cause of infertility and adverse pregnancy outcomes [2].

CT infection, like STIs in general, is primarily a woman's health issue since the manifestations and consequences are more damaging to the reproductive health of women than men. Based on the available evidence, $15 \%-20 \%$ of women with CT lower genital tract infection develop PID; approximately $4 \%$ develop chronic pelvic pain, $3 \%$ infertility and $2 \%$ adverse pregnancy outcome $[3,4]$. However, a considerable proportion of CT $(25 \%-35 \%$ of infections in men and $65 \%-75 \%$ in women) remain asymptomatic and may escape treatment. Subsequent complications still result in substantial morbidity and high costs for health care due to severe adverse effects on reproductive health $[1,5]$.

The prevalence and the risk factors of STIs in women differ by country and setting and by the existence of symptoms $[3,6-8]$.
Reports have shown that risk factors for CT infection are: age 18-27 years, non-use of contraceptives, frequent post-coital bleeding, painful micturition, urban residence, low income, cervical infection, polygamous marriage and mucopurulent cervical discharge $[1,9,10]$.

In order to prevent and control CT infection, there is a need to identify women with asymptomatic or mild endocervical infections and those at increased risk for acquisition of this infection. However, there is little information concerning the prevalence and risk factors for $\mathrm{CT}$ in Mazandaran province, northern Islamic Republic of Iran. Therefore, we aimed to investigate the prevalence and associated demographic, behavioural and clinical risk factors of CT in women attending the gynecology unit of health centres in Babol city.

\section{Methods}

There are 15 health care centres of Babol University of Medical Sciences. The study was carried out among women attending 6 of these centres selected by cluster sampling. Babol city was divided into 3 areas and 2 health care centres were selected from each area. Thus, assuming a prevalence of 0.1 and marginal rate of error of $0.025,550$ women were prospectively enrolled during the period 2003-2004 by an expert midwife in every centre. Inclusion criteria were: women $15-45$ years who were sexually active and had come to the gynaecology units in these centres. They could be either symptomatic (vaginal discharge, genital ulcer and lower abdominal pain) or asymptomatic (routine examination). Exclusion criteria were: antibiotic use within the past 4 weeks, pregnancy or abnormal uterine bleeding to exclude certain confounding factors. 
A standardized questionnaire was completed by one of the authors for each woman, followed by a pelvic examination. The questionnaire included the following information:

- sociodemographic variables, including age, educational and professional status, marital status and income;

- behavioural variables including age at starting sexual activity, having extramarital sex, perception of the husband having extramarital sex in the previous 3 months, history of sexual abuse, drug addiction, cigarette and alcohol use of the woman and her husband, current type of contraception used, use of condoms, having ever heard of STI and HIV, history of STI and PID and their frequency;

- clinical variables including reasons for presentation at the clinic, chief complaint, data recorded by physical examination, number of pregnancies, abortions, infertility.

Blood samples were taken in sterile disposable syringes from all the women and delivered to a single laboratory where all the tests were performed. C. trachomatis was diagnosed using an ELISA test. Briefly, serum was separated from clear nonhaemolytic non-icteric and non-lipemic samples. Chlamydia IgA and IgG were detected by standard kits (Trinity Kits, Trinity, United States of America).

The Medical Ethics Committee of the university approved the study and informed consent was obtained from all participants before their enrolment.

Results are presented as percentages and comparison of the results was performed by the chi-squared test and Fisher exact twotailed test where appropriate. All tests were two-sided and the level of significance was set at $P<0.05$. The diagnostic performance of clinical symptoms was evaluated us- ing for sensitivity, specificity and positive and negative predictive values. Statistical analysis was performed using the SPSS, version 11 .

\section{Results}

The prevalence of CT among our sample was $11.6 \%(64 / 550)$. All the women were married and their mean age was 32.3 years, $44.9 \%$ of the women and $39.3 \%$ of their husbands had at least a primary education (25.1\% of the women and $26.7 \%$ of their husbands had high-school diplomas, and only $7.5 \%$ of the women and $12.4 \%$ of their husbands had a university degree). The majority of the women were housewives, and $40.9 \%$ of their husbands were tradesmen. Regarding the economic situation, 38.4\% had a household income of $<$ US\$ 116.3 per month.

The mean age at first sexual intercourse was 19.02 years. None of the women reported having sex with more than 1 partner, extramarital sex or a history of sexual abuse. Most of the women (95\%) were not aware if their husbands had extramarital sexual relations but $2.9 \%$ reported that their husbands were drug addicts and $30.1 \%$ said their husbands smoked cigarettes. The commonest contraceptive method used was withdrawal and then tubal ligation $(29.5 \%$ and $21.8 \%$ respectively); $76.4 \%$ of the participants had never used condoms.

The main reason for attendance at the gynaecology clinics was urogenital complaints $(59.3 \%)$; the most frequent complaints were dyspareunia $(34.3 \%)$ and vaginal discharge $(26.7 \%)$. The most frequent signs were cervix ectopy $(18 \%)$ and lower abdominal tenderness $(16.9 \%)$ and the most frequent diagnosis was cervicitis $(24.5 \%)$. On physical examination cervical motion tenderness was present in $6 \%$ of the women, while 
uterine/adnexal tenderness was noted in $11.1 \%$.

Mean gravidity and abortion were 2.53 and 0.32 respectively, and $22 \%$ of the women had a history of PID and $7.6 \%$ a history of infertility. All the women denied having a past history of STIs although they did not know STI symptoms or they thought they were not important, or felt shy to visit the health centres. In fact, $85.3 \%$ of them had not heard about STIs, while $93.8 \%$ had heard of HIV.

Table 1 shows the demographic and behavioural variables significantly associ- ated with $\mathrm{CT}$ infection. The association of urogenital manifestations with $\mathrm{CT}$ infection is shown in Table 2.

There was a significant association between woman's age ( $<25$ years) and CT infection; age $<25$ years was associated with a significantly higher risk of CT infection. In addition, low income ( $<$ US\$ 116.3 per month) and husband's low educational level were significantly positively associated with CT infection $(P=0.04)$. There was also a significant positive association between CT infection and husband's drug addiction and a negative association with condom use.

\begin{tabular}{|c|c|c|c|c|c|c|c|}
\hline \multirow[t]{2}{*}{ Risk factor } & \multicolumn{2}{|c|}{ Positive } & \multicolumn{2}{|c|}{ Negative } & \multicolumn{2}{|c|}{ Total } & \multirow[t]{2}{*}{$P$-value } \\
\hline & No. & $\%$ & No. & $\%$ & No. & $\%$ & \\
\hline Husband's education & & & & & & & $<0.04$ \\
\hline Illiterate/primary school & 37 & 15.5 & 202 & 84.5 & 239 & 100 & \\
\hline High-school diploma & 20 & 8.2 & 223 & 91.8 & 243 & 100 & \\
\hline University education & 7 & 10.3 & 61 & 89.7 & 68 & 100 & \\
\hline Women's age (years) & & & & & & & $<0.01$ \\
\hline$\leq 25$ & 19 & 20.2 & 71 & 79.8 & 89 & 100 & \\
\hline $26-35$ & 25 & 9.3 & 243 & 90.7 & 268 & 100 & \\
\hline $36-45$ & 20 & 10.4 & 173 & 89.6 & 193 & 100 & \\
\hline Condom use & & & & & & & $<0.05$ \\
\hline Mostly/sometimes & 11 & 8.5 & 119 & 91.5 & 130 & 100 & \\
\hline Never & 53 & 12.6 & 367 & 87.4 & 420 & 100 & \\
\hline Addiction of husband & & & & & & & $<0.02$ \\
\hline Yes & 5 & 31.2 & 11 & 68.8 & 16 & 100 & \\
\hline No & 59 & 11.0 & 475 & 89.0 & 534 & 100 & \\
\hline $\begin{array}{l}\text { Ever heard of sexually } \\
\text { transmitted infections }\end{array}$ & & & & & & & $<0.04$ \\
\hline Yes & 4 & 4.9 & 77 & 95.1 & 81 & 100 & \\
\hline No & 60 & 12.8 & 409 & 87.2 & 469 & 100 & \\
\hline Ever heard of HIV & & & & & & & $<0.003$ \\
\hline Yes & 54 & 10.5 & 462 & 89.5 & 516 & 100 & \\
\hline No & 10 & 29.4 & 24 & 70.6 & 34 & 100 & \\
\hline Income (US\$/month) & & & & & & & $<0.04$ \\
\hline$<116.3$ & 31 & 14.7 & 180 & 85.3 & 211 & 100 & \\
\hline $116.3-<232.5$ & 26 & 10.8 & 214 & 89.2 & 240 & 100 & \\
\hline $232.5-348.8$ & 1 & 1.7 & 57 & 98.3 & 58 & 100 & \\
\hline$>348.8$ & 6 & 14.6 & 35 & 85.4 & 41 & 100 & \\
\hline
\end{tabular}




\begin{tabular}{|c|c|c|c|}
\hline \multirow[t]{2}{*}{ Urogenital manifestations } & \multirow[t]{2}{*}{ Total no. } & \multicolumn{2}{|c|}{$\begin{array}{c}\text { Positive for } \\
\text { C. trachomatis }(n=64)\end{array}$} \\
\hline & & No. & $\%$ \\
\hline \multicolumn{4}{|l|}{ Symptoms } \\
\hline Vaginal discharge & 147 & 19 & 12.9 \\
\hline Frequent post-coital bleeding & 18 & 3 & 16.7 \\
\hline Lower abdominal pain & 121 & 17 & 14.0 \\
\hline Genital itching & 85 & 14 & 16.5 \\
\hline Difficulty in urination & 52 & 5 & 9.6 \\
\hline Dyspareunia & 189 & 19 & 10.1 \\
\hline Menstrual irregularities & 92 & 12 & 13.0 \\
\hline \multicolumn{4}{|l|}{ Signs } \\
\hline Genital sores/ulcers & 0 & 0 & 0.0 \\
\hline Pruritus & 16 & 3 & 18.8 \\
\hline Cervical friability* & 73 & 23 & 31.3 \\
\hline Cervical ectopy & 99 & 9 & 9.1 \\
\hline Redness of vulva or vagina & 27 & 5 & 18.5 \\
\hline Mucopurulent cervicitis* & 29 & 8 & 27.6 \\
\hline Lower abdominal tenderness & 93 & 12 & 12.9 \\
\hline Rebound in the lower abdomen & 34 & 4 & 11.8 \\
\hline Cervix motion tenderness & 33 & 3 & 9.1 \\
\hline Uterine/adnexa tenderness & 61 & 6 & 9.8 \\
\hline Discharge & 489 & 12 & 2.4 \\
\hline
\end{tabular}

*Significant at $\mathrm{P}<0.05$.

Not ever having heard of STIs or HIV was also significantly associated with CT infection. The only signs significantly associated with $\mathrm{CT}$ infection were cervical friability and mucopurulent cervicitis. There were no significant associations with any other variables studied.

Syndromic diagnosis of CT infection had moderate sensitivity of $64 \%$ but a poor positive-predictive value (20.4\%) (Table 3).

\section{Discussion}

The prevalence of CT infection reported in this study $(11.6 \%)$ is moderate in comparison with the low and high rates reported by other studies ( $2.5 \%$ and $47 \%$ respectively) in similar study subjects and settings [2,1115]. However, it is worth mentioning that the diagnostic tests varied in the different studies and could contribute to the differences in rates across countries. ELISA used in this study is less sensitive than polymer-

Table 3 Diagnostic performance of syndromic diagnosis for Chlamydia trachomatis infection

\begin{tabular}{lcccc}
\hline $\begin{array}{l}\text { Results of the } \\
\text { syndromic } \\
\text { diagnosis }\end{array}$ & \multicolumn{2}{c}{$\begin{array}{c}\text { Results of laboratory test } \\
\text { Positive }\end{array}$} & $\begin{array}{c}\text { Nogative } \\
\text { No. }\end{array}$ & $\begin{array}{c}\text { No. } \\
\text { No. }\end{array}$ \\
\hline Positive & 32 & 20.3 & 125 & 79.6 \\
Negative & 18 & 10.2 & 159 & 89.8 \\
\hline
\end{tabular}

Sensitivity: $64.0 \%$; specificity: $56.0 \%$; positive predictive value: $20.4 \%$; negative predictive value: $89.8 \%$. 
ase chain reaction (PCR) and cell culture but more sensitive than direct fluorescence assay. In studies conducted in low prevalence areas, PCR, indirect fluorescence assay, ligase chain reaction and cell culture were used. In higher prevalence areas, PCR + strand displacement amplification, DNA probe, ELISA, direct fluorescence assay + culture were used.

Studies conducted in the Islamic Republic of Iran are very limited. In 1 study in the north of the country, Sari city (which is near Babol), the prevalence of CT in pregnant women was $2.75 \%$ which is lower than our finding [16]. Other reports from STI clinics or only in symptomatic women $(2.3 \%-12 \%)$ are also lower than our result which suggests that the CT prevalence obtained in our study is relatively high $[1,17]$. This could be attributed to treatment practices in different areas of the country. Because of socioeconomic reasons, women attending gynecology clinics might not have consulted a doctor and might be seeking treatment for the first time at those clinics for health problems. Thus the likelihood of diagnosing the infection at the clinic is higher. This may explain the significant association between positive CT and income or husband's education found in our study. Mild or asymptomatic disease course, absence of partner notification and subsequent lack of follow-up treatment for CT in sexual partners may also contribute to high prevalence of CT in our study.

In an attempt to identify high-risk women referred to the clinics, we studied several determinants. We found that woman's age, husband's education and household income were significantly associated with positive CT. The significant association of education and income with positive $\mathrm{CT}$ is consistent with some studies $[1,16]$. Similarly, the significant association between young age $(<25$ years) and CT concurred with others studies [16,18].
The decreased susceptibility to infection with age has been attributed to epithelial changes, which may result in a decreased rate of infection in the elderly [19]. Partial immunity can also prevent re-infection in the elderly [20]. The effect of age may also be attributed to residual confounding due to non-measured sexual characteristics such as frequency of sexual intercourse per partner and the duration of intercourse [1].

Regarding behavioural markers, only the drug addiction of husbands and not ever having heard of STIs or HIV were positively associated with CT infection [21]. Regarding sexual behaviour, not surprisingly, most women answered negatively to most of the questions. This is probably due to strong religious beliefs in the community about extramarital sex. Such behaviour by husbands is likely of course to occur in secret and their wives would be unaware of it. Obtaining information on such issues is almost impossible in our community because such activities are often illegal, and lack of such data is a limitation of our study to determine an association between sexual activity and CT. However, we failed to find an association between PID and CT infection.

In agreement with other studies, addiction of men was independently associated with positive CT [17,19-22] while condom use was negatively associated. At the same time, several independent predictors of CT infection reported by others as useful components of screening strategies were not identified in our study. For example, reasons for visit and history of PID were not identified as risk factors; this might be attributed to uniformity of these factors in the women in our study.

ELISA, which was used to detect CT, is less sensitive than nucleic acid amplified tests and thus our estimate probably underestimates the true prevalence of CT and the 
use of more precise techniques would have given a higher prevalence.

The prevalence of STIs in our community, especially in the general population at risk, is not known and although ours was not a population-based study, it is doubtful whether our results were affected by excluding patients attending other health care providers, such as general practitioners and obstetric units. However, in order to obtain a complete picture of CT infection in our community, we need to consider the wider population with regard to age, sexual activity, clinical presentation and STI status.

Using ELISA as the gold standard, syndromic diagnosis proved to be an unreliable test with only moderate sensitivity and poor positive predictive value, which casts doubts on the validity of this approach for CT management.

In conclusion, CT infection seems widespread among the sexually active general population of our country. These observations imply that, as well as a sexual health promotion policy for controlling STIs, specific preventive and intervention strategies for CT should be developed and targeted among the sexually active general population. These include promotion of community awareness about STIs by the media and the establishment of STI clinics free of charge. Additionally, because sexual activity in men is probably less limited in comparison with that in woman, women are often infected through their male partners. Because of social barriers, high-risk sexual behaviour in our community is not recognized or admitted. Thus if we do not address this situation, STIs will remain unknown and under-diagnosed and -treated in the community and may develop into a real public health problem.

\section{Acknowledgements}

This research received technical and financial support jointly from the Division of Communicable Diseases and the Small Grants Scheme for Operational Research in Tropical and Other Communicable Diseases of the World Health Organization Regional Office for the Eastern Mediterranean.

We thank Dr K. Kamali of the STI/AIDS Programme of Disease Management for his assistance in the assessment and approval of this project. We also thank Dr Mohammadpoor who helped with the statistical analysis. We are also grateful to all the midwives and clerical staff of the health centres for their cooperation in the study and to Miss Akbarzadeh Pasha for her help in data collection and assistance in preparation and editing of the article.

\section{References}

1. Van Duynhoven YT et al. Different demographic and sexual correlates for chlamydial infection and gonorrhoea in Rotterdam. International journal of epidemiology, 1997, 26(6):1373-85.

2. Wilkinson $D$ et al. Unrecognized sexually transmitted infections in rural South African women: a hidden epidemic. Bulletin of the World Health Organization, 1999, 77(1):22-8.

3. Johnson RA. Diagnosis and treatment of common sexually transmitted disease in women. Clinical cornerstone, 2000, 3(1):1-11.

4. Paavonen J, Eggert-Kruse W. Chlamydia trachomatis: impact on human reproduction. Human reproduction update, 1999, 5(5):433-47.

5. Crotchfelt KA et al. Detection of Neisseria gonorrhoeae and Chlamydia trachomatis in genitourinary specimens from men and women by a coamplification PCR assay. Journal of clinical microbiology, 1997, 35(6):1536-40. 
6. Dowe $\mathrm{G}$ et al. High prevalence of genital chlamydia trachomatis infection in women presenting in different clinical settings in Jamaica: implications for control strategies. Sexually transmitted infections, 1999, 75:412-6.

7. Armando Brito de SA et al. Genital infection by Chlamydia trachomatis in Lisbon: prevalence and risk markers. Family practice, 2002, 19:362-4.

8. Cerkez-Habek J, Habek D. Epidemiologic characteristics of sexually transmitted infection/coinfection with Chlamydia trachomatis and Neisseria gonorrhoeae. Acta medica Croatica, 2001, 55(4-5):191201.

9. Guaschino S, De Seta F. Update on Chlamydia trachomatis. Annals of the New York Academy of Sciences, 2000, 900:293-300.

10. Singh $V$ et al. Polymerase chain reaction for detection of endocervical Chlamydia trachomatis infection in women attending a gynecology outpatient department in India. Acta cytologica, 2002, 46(3):540-4.

11. Tchoudomatis $K$, Nuhov $P$, Tchapanova A. Prevalence, epidemiological and clinical correlates of genital Chlamydia trachomatis infection. Journal of the European Academy of Dermatology and Venereology, 1998, 11 (3):214-20.

12. Claesys $P$ et al. Sexually transmitted infections and reproductive health in Azerbaijan. Sexually transmitted diseases, 2001, 28(7):372-8.

13. Steen $\mathrm{R}$ et al. Evidence of declining STD prevalence in a South African mining community following a core-group intervention. Sexually transmitted diseases, 2000, 27(1):1-8.

14. Hardick J et al. Surveillance of Chlamydia trachomatis and Neisseria gonorrhoeae infection in women in detention in Baltimore, Maryland. Sexually transmitted diseases, 2003, 30(1):64-70.
15. Taffa $\mathrm{N}$ et al. Prevalence of gonococcal and Chlamydia infections and sexual risk behavior among youth in Addis Ababa, Ethiopia. Sexually transmitted diseases, 2002, 29(12):828-33.

16. Behrozi R, Badami N. Assessment of prevalence of chlamydial infection in women pregnant referring the hospitals of Tehran University of Medical Sciences in 1994 years. Journal of Mazandaran University of Medical Sciences, 1999, 22-23 Spring-Summer:26-31.

17. Claeys $P$ et al. Prevalence and risk factors of sexually transmitted infections and cervical neoplasia in women's health clinics in Nicaragua. Sexually transmitted infections, 2002, 78(3):204-7.

18. Kissin DM et al. Epidemiology and natural history of ligases chain reaction detected chlamydial and gonococcal infections. Sexually transmitted infections, 2002, 78(3):208-9.

19. Latino MA et al. Infezioni cervico-vaginali. Studio sulla popolazione dell'area torinese [Cervico-vaginal infections. Study of a population in the Turin area]. Minerva ginecologica, 2002, 54(4):309-16.

20. Bachmann LH et al. Prevalence and factors associated with gonorrhea and chlamydial infection in at-risk females presenting to an urban emergency department. Sexually transmitted diseases, 2003, 30(4):335-9.

21. Passey $M$ et al. Community based study of sexually transmitted diseases in rural women in the highlands of Papua New Guinea: prevalence risk factors. Sexually transmitted infections, 1998, 74(2):120-7.

22. Paris $M$ et al. Prevalence of gonococcal and chlamydial infections in commercial sex workers in a Peruvian Amazon city. Sexually transmitted diseases, 1999, 26(2):103-7. 